\title{
Truth seekers in opinion dynamics models
}

\author{
Krzysztof Malarz ${ }^{1,2, *}$ \\ ${ }^{1}$ Faculty of Physics and Applied Computer Science, AGH University of Science and Technology, \\ al. Mickiewicza 30, PL-30059 Kraków, Euroland \\ ${ }^{2}$ Institute of Theoretical Physics, University of Cologne, D-50923 Köln, Euroland
}

\begin{abstract}
We modify the model of Deffuant et al. to distinguish true opinion among others in the fashion of Hegselmann and Krause (http://jasss.soc.surrey.ac.uk/9/3/10.html). The basic features of both models modified to account for truth seekers are qualitatively the same.
\end{abstract}

Keywords: Monte Carlo simulation, sociophysics, opinion dynamics

Introduction. Opinion dynamics simulations 1.2.3.4 (see Ref. 5 for review) seem to be the most fashionable part of sociophysics ${ }^{6}$. When your girlfriend asks you 'Do I look fine?' you may use the Sznajd mode ${ }^{2}$ to answer this general question as Sznajd agents behave similarly to magnetic spins: they are allowed to have only two opinions $s_{i} \in\{0,1\}$ — for example 'yes' or 'no'. But when the question is open - for instance: 'How am I looking like today?' - the space of possible answers enlarges drastically. The correct answers are 'Super!' and 'Exceptionally!' but not 'O.K.' or even 'nice'. The latter is reserved for the same question but about a pretty girl starring in any Hollywood movie. For modeling such opinions the Hegselmann-Krause ${ }^{3}$ (H-K) or Deffuant et $a l^{\underline{4}}$ models are more appropriate as they offer a continuous interval of possible opinions $s_{i} \in[0,1]$.

Now, you and your interlocutor may have a wrong opinion about your girlfriend. The truth may be elsewhere. The modifications of $\mathrm{H}-\mathrm{K}$ model which allow to introduce truth seekers among exchanging their opinions agents were presented very recently in Ref. 7 .

In this paper we would like to check if the same is available for Deffuant et al. mode ${ }^{\underline{4}}$ were Assmann ${ }^{8}$ had already introduced a multitude of truths.

The model. In the original Deffuant et al. model two persons (let say $i$ and $j$ ) exchange their opinion about given topic if their current opinions do not differ more than confidence level $\varepsilon$, i.e. when $\left|s_{i}-s_{j}\right| \leq \varepsilon$. In such case, after discussion their change their opinions slightly, i.e.

$$
\left\{\begin{array}{l}
s_{i} \rightarrow s_{i}+\mu \delta \\
s_{j} \rightarrow s_{j}-\mu \delta
\end{array}\right.
$$

where $\delta=s_{j}-s_{i}$ and $\mu \in[0,1 / 2]$ describes a speed of opinion changes. If their opinions are too distant, i.e. $|\delta|>\varepsilon$, the agents do not change their opinions at all.

To account for the true opinion Hegselmann and Krause introduced two additional parameters: $T \in[0,1]$ and $\alpha_{i}$ - which represent the true opinion and the strength of the attraction to the truth for $i$-th agent, respectively ${ }^{\text {? }}$.

With these two additional terms Eq. (11) for the Deffuant et al. model becomes

$$
\left\{\begin{array}{l}
s_{i} \rightarrow s_{i}+\mu\left[\alpha_{i}\left(T-s_{i}\right)+\left(1-\alpha_{i}\right) \delta\right] \\
s_{j} \rightarrow s_{j}+\mu\left[\alpha_{j}\left(T-s_{j}\right)-\left(1-\alpha_{j}\right) \delta\right]
\end{array} .\right.
$$

The case $\alpha_{i}=0(i=1, \cdots, N)$ corresponds to the original Deffuant et al. model and for $\alpha_{i}=1(i=1, \cdots, N)$ agents do not exchange opinions each to other but tends towards the true one.

The results. In Fig. 1 the results of simulation are presented for opinion dynamics of $N=500$ agents with initially randomly chosen opinions $s_{i}$ (the same for all sub-figures). The model parameters are shown in the headline of all sub-figures. In Fig. 1(a) the opinion dynamics governed by original Deffuant et al. model is presented $\left(\alpha_{i}=0\right.$ for all $\left.i\right)$. In Fig. प(b) all agents are the truth seekers. In Figs. 1(c)-(e) half of agents is the truth seeker $\left(\alpha_{i}>0\right.$, marked as green) while the second half is not $\left(\alpha_{i}=0\right.$, marked as red). In Fig. 团(f) only $2 \%$ agents search for the truth. Parts c/d, c/e, c/f differ only with $T, \varepsilon$ and the fraction of the truth seekers, respectively. For $N=10^{2}$ and $10^{3}$ the results are qualitatively the same.

The obtained results support the observation from Figs. $1-8$ in Ref. 7 .

In conclusions, the Deffuant et al. mode ${ }^{4}$ with necessary modifications which allow to simulate true opinion among others gives qualitatively the same results as the $\mathrm{H}-\mathrm{K}$ model modified for the same purpose $\mathrm{F}^{7}$.

Acknowledgments. Author is grateful to Dietrich Stauffer for his hospitality in Köln and to EU grant GIACS. Part of calculation was carried out in ACKCYFRONET-AGH. Time on HP Integrity Superdome is financed with grant no. MNiI/HP_I_SD/AGH/047/2004.

\footnotetext{
* URL: http://home.agh.edu.pl/malarz/ Electronic address: malarz@agh.edu.pl

${ }^{1}$ D. Stauffer, M. Sahimi, Physica A364, 537 (2006); T. Car-
}

letti, D. Fanelli, S. Grolli, A. Guarino, Europhys. Lett. 74, 222 (2006); A. Pluchino, V. Latora, A. Rapisarda, Eur. Phys. J. B50, 169 (2006); C.M. Bordogna, E.V. Albano, 
(a) $\mathrm{N}=500, \varepsilon=0.07, \mu=0.05, \mathrm{~T}=0.25, \alpha=0(100 \%)$

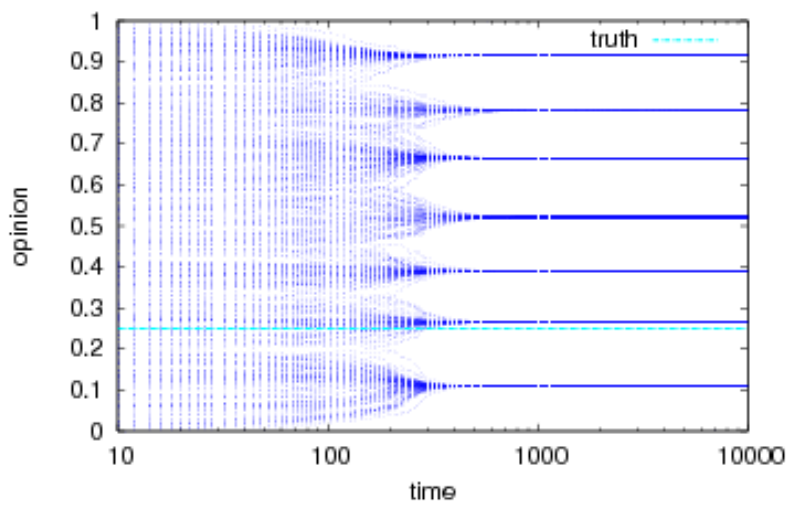

(c) $N=500, \varepsilon=0.1, \mu=0.15, T=0.5, \alpha=0.1(50 \%)$

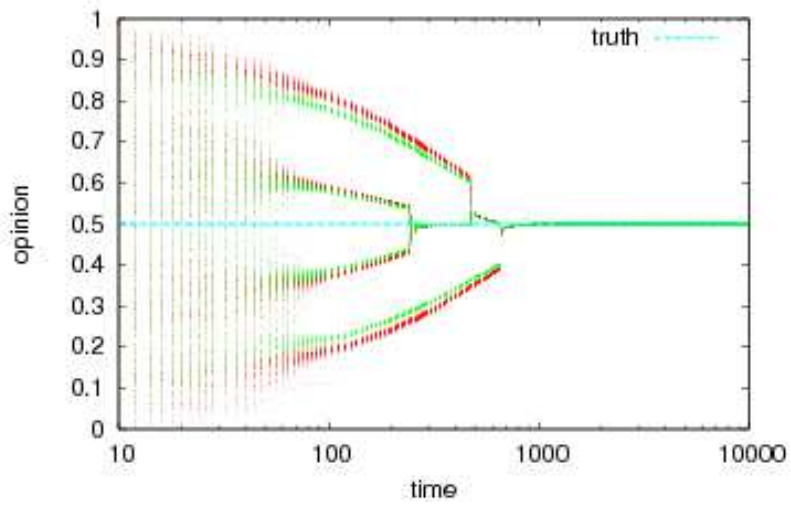

(e) $\mathrm{N}=500, \mathrm{E}=0.05, \mu=0.15, \mathrm{~T}=0.5, \alpha=0.1(50 \%)$

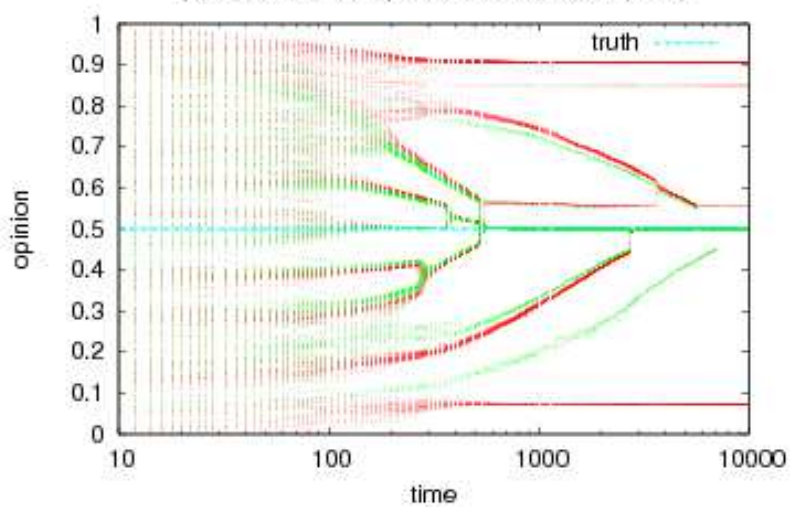

(b) $\mathrm{N}=500, \mathrm{e}=0.07, \mu=0.05, \mathrm{~T}=0.25, \alpha=0.15(100 \%)$

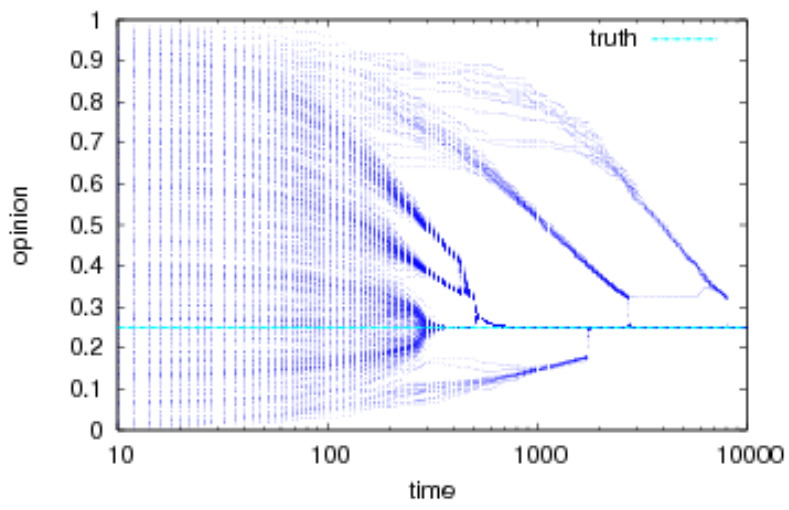

(d) $N=500, E=0.1, \mu=0.15, T=0.05, \alpha=0.1(50 \%)$

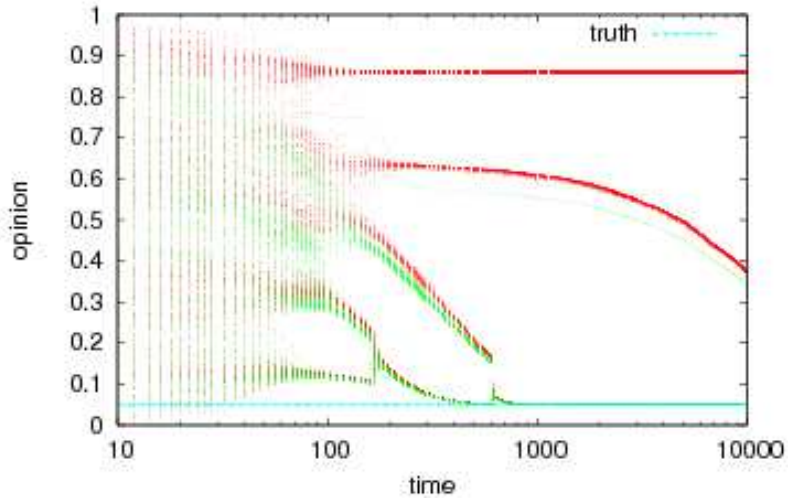

(f) $\mathrm{N}=500, \varepsilon=0.1, \mu=0.15, \mathrm{~T}=0.5, \alpha=0.1(2 \%)$

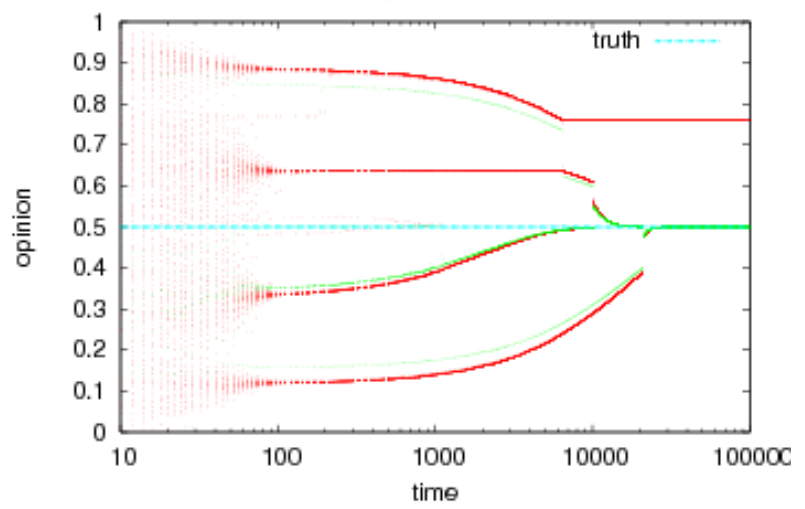

FIG. 1: (Color online). A few examples of opinion dynamics for Deffuant model with truth seekers. The model parameters and given in sub-figures captions. The straight horizontal line indicates the 'truth'.

Int. J. Mod. Phys. C17, 409 (2006); S. Gekle, L. Peliti, S. Galam, Eur. Phys. J. B45, 569 (2005).

2 K. Sznajd-Weron, Acta Phys. Pol. B36, 2537 (2005); K. Sznajd-Weron, J. Sznajd, Int. J. Mod. Phys. C11, 1157 (2000).

3 R. Hegselmann, U. Krause, J. Artif. Soc. Soc. Simul. 5, (2002) http://jasss.soc.surrey.ac.uk/5/3/2.html

4 G. Deffuant, F. Amblard, G. Weisbuch, T. Faure, J. Artif. Soc. Soc. Simul. 5, (2002) http://jasss.soc.surrey.ac.uk/5/4/1.html
${ }^{5}$ R. Toral, C.J. Tessone, to appear in Commun. Comput. Phys.; D. Stauffer, AIP Conf. Proc. 779, 56 (2005).

6 D. Stauffer, S. Moss de Oliveira, P.M.C. de Oliveira, J.S. Sá Martins, Biology, Sociology, Geology by Computational Physicists, Elsevier, Amsterdam (2006).

7 R. Hegselmann, U. Krause, J. Artif. Soc. Soc. Simul. 9, (2006) http://jasss.soc.surrey.ac.uk/9/3/10.html

8 P. Assmann, Int. J. Mod. Phys. C15, 1439 (2004). 